DOI: 10.12731/2070-7568-2020-2-65-77

УДК 338.27

\title{
ОГРАНИЧЕНИЯ МОДЕЛЕЙ ОБЩЕГО РАВНОВЕСИЯ ПРИ ПРОГНОЗИРОВАНИИ ДИНАМИКИ СОЦИАЛЬНО-ЭКОНОМИЧЕСКИХ СИСТЕМ
}

\author{
Голубев Ф.С.
}

В статье рассматриваются ограничения возникающие при прогнозировании динамики социально-экономических систем на основе моделей общего равновесия. Автор рассматривает различные вариации данных моделей, возможности и критические моменты, выявленные за время их использования. В статье предлагается обратить внимание на поведенческий подход при разработке прогнозов применительно к социально-экономической среде для приближения прогнозных результатов к реальной экономике и учете влияния более глубоких прочессов микроуровня на показатели макроуровня.

Цель - выявить ограничения различного рода моделей на основе таблии «затраты-выпуск» при прогнозировании динамики сочиально-экономических систем.

Метод и методология проведения работы: работа основывается на методах сравнительного анализа методологий прогнозирования на основе различных вариащий моделей общего равновесия.

Результаты: полученные результаты представляют собой теоретическую основу для использования такого вида анализа применительно к другим методам прогнозирования.

Область применения результатов: полученные в статье результаты применимы к использованию прогнозов к выявлению изменений социильно-экономических систем.

Ключевые слова: сочиально-экономический прогноз; модели на основе таблии «затраты-выпуск»; гравитационные модели; экономико-психологические факторы; методология прогнозирования. 


\section{RESTRICTIONS OF MODELS OF GENERAL EQUILIBRIUM IN FORECASTING THE DYNAMICS OF SOCIO-ECONOMIC SYSTEMS}

\section{Golubev F.S.}

The dynamics of forecasts of dynamics and socio-economic systems based on general equilibrium. The author considers various variations of these models, opportunities and critical points identified for their use. The article proposes to pay attention to a reasonable approach when developing forecasts in relation to the socio-economic environment in order to bring the predicted results closer to the real economy and to study the influence of deeper micro-level processes on macro-level indicators.

Purpose - identify the limitations of various kinds of models based on "input-output" tables when forecasting the dynamics of socio-economic systems.

Methodology: the work is based on the methods of comparative analysis of forecasting methodologies based on various variations of the general equilibrium models.

Results: The obtained results represent the theoretical basis for using this type of analysis in relation to other forecasting methods.

Practical implications: The results obtained in the article are applicable to the use of forecasts to identify changes in socio-economic systems.

Keywords: socio-economic forecast; models based on input-output tables; gravity models; economic and psychological factors; forecasting methodology.

Прогнозирование социально-экономического развития выступает одной из основных функций современной экономической науки. Согласно позиции Нобелевского лауреата по экономике М. Фридмана, именно возможность адекватного прогноза является критерием истинности теоретических построений [14]. Между тем, по-видимому, все время существования данной отрасли знания качество предска- 
зания оставляло желать лучшего. Традиционно проблемы с предвидением динамики и направления экономических процессов критики относили на счет низкого качества методов прогнозирования.

Однако, может быть и вторая сторона проблемы. Существующий уровень и качество прогнозов в последние тридцать лет демонстрируют более существенное, чем раньше расхождение между тем, что ожидалось и реальным развитием событий. Рост неопределенности приводит к усложнению факторов и связей, имеющих существенное значение для предсказания будущего. Данный рост идет сразу по двум направлениям. С одной стороны, развитие научных знаний об устройстве экономики, социальных процессах, психологических компонентах поведения экономических агентов требует учета все большего количества переменных. С другой стороны, само экономическое развитие на каждом новом витке становится все более сложной системой, в которой неоправданное абстрагирование от каких-либо факторов, существенно снижает прогностические возможности применяемых методов. Наиболее существенными из факторов, которые определяют социально-экономическое развитие сегодня по мнению ряда исследователей выступают поведенческие или экономико-психологические факторы [7, 11 и др.].

Попробуем проанализировать возможности существующих методов прогнозирования через призму этих двух критериев: а) через методологическую релевантность метода прогнозирования; б) через соответствие уровня комплексности метода прогнозирования реальной сложности экономической системы.

Для начала определимся с самим понятием прогноза в экономической науке. Слово «прогноз» происходит от греческого «prognosis», что означает «предузнавание». Согласно толковому словарю Ожегова, прогноз представляет собой предсказание о развитии и исходе каких-либо событий, явлений на основании имеющихся данных [10]. Определения прогноза в экономических словарях отличает наличие научно-обоснованной гипотезы в качестве базы, на которой строится прогноз [2]. Результатом прогноза выступает вероятностная оценка состояния системы и состояния объектов, от которых зави- 
сит данная система, что, на наш взгляд, говорит о том, что любой прогноз подразумевает комплексный научно-обоснованный анализ прогнозируемых изменений системы, анализ взаимосвязей объектов с другими объектами внутри системы, определение степени влияния каждого объекта на систему в целом.

На сегодняшний день накоплен довольно большой опыт в построении социально-экономических прогнозов с использованием различных методов. Самым распространенным из них $[9,1]$, повидимому, является метод построения моделей общего равновесия на основе таблиц «затраты-выпуск» [5] или модели межотраслевого баланса. Таблицы «затраты-выпуск» представляют собой разделение экономики на сектора по сферам производства и отражают взаимодействие между ними. Особенности присутствия на рынке иностранного сектора выражаются отдельной производственной отраслью, а домашнее хозяйство и государственный сектор представляются крупными непроизводственными секторами. Стоит отметить, что в государственный сектор входят лишь сферы государственного управления, не занятые в производстве. Вся произведенная продукция выражается в единице обмена и может быть представлена любым товаром, но чаще всего за единицу обмена принимается доллар, причем в его относительном стоимостном выражении, что требует введения в методологию прогноза базового периода расчетов. Взаимосвязи между секторами внутри таблицы выражаются линейными зависимостями, тем самым отражая динамику показателей от одного сектора к другому. Модели на основе таблиц «затраты-выпуск» (модели общего равновесия или межотраслевые модели) демонстрируют построение баланса, распределения различных видов экономических благ между производителями и потребителями. Таким образом рассматривая экономику стран ее можно представлять в виде системы взаимосвязанных видов экономической деятельности [5].

История разработки и использования балансовых моделей на основе таблиц «затраты-выпуск» в нашей стране развивалась от баланса народного хозяйства 1924 г., до системы национальных 
счетов, используемых в России с 1995 г. Мировая история системы национальных счетов началась в 1953 г. в США и Европе ввиду отсутствия единой информационной базы для построения мировых прогнозов и проблемы учета данных по странам [6].

За время своего существования модели межотраслевого баланса получили некоторые вариации, например, для анализа взаимовлияния экономик между странами используют узкоспециализированный метод, который называется межстрановой межотраслевой баланс [13], демонстрирующий анализ торговли между странами и взаимовлияние экономик друг на друга, где иностранный сектор рассматривается в каждой из сфер, а не представляется отдельной отраслью, как в «классических» моделях.

Другой разновидностью такого рода моделей являются динамические межотраслевые модели, где один из показателей является динамично изменяемым, тем самым исследователи имитируют развитие системы, через воздействие на определенный компонент. Так, например, в работе по использованию динамических межотраслевых моделей [1] показан учет инвестиций в человеческий капитал и значимость этого параметра при долгосрочных прогнозах развития РФ, исходя из показателей ВВП (т.е. динамическим параметром выступает человеческий капитал, влияя на него авторы получили различные прогнозные показатели ВВП). Выявленная регрессионная взаимосвязь между инвестициями в человеческий капитал и ВВП позволила построить авторам модель исходя из трех возможных сценариев развития для страны на период до 2045 г. [1]. Полученные результаты авторы сравнивали с результатами аналогичного исследования [9], обосновав схожие результаты взаимосвязью между человеческим капиталом и ВВП. На наш взгляд разработанная модель демонстрирует уровень значимости инвестиций в человеческий капитал для развития страны, но при этом слабым местом данной модели является статичный математический прогнозный аппарат, с высоким уровнем абстракции от реально протекающих процессов во внутренней экономике. К тому же разного объема инвестиции в человеческий капитал имея регрессионную взаимосвязь не описы- 
вают сложности внутренних процессов, вкладываемых в данную сферу (как осуществляются инвестиции, на что именно они влияют, как воспринимают их агенты микроуровня), отмечается лишь увеличение финансирования той или иной сферы формирующей человеческий капитал (образование, здравоохранение, культура), но не описывается работоспособность данного капитала. Таким образом, по выделенным выше критериям получается, что на методологическом уровне не обеспечивается переход от агентов микроуровня к макроэкономической динамике. На уровне комплексности теряется факт реагирования экономических агентов на изменения.

Другой распространенный метод прогнозирования, относящийся к моделям общего равновесия, получил название гравитационных моделей. Данный метод сочетает в себе элементы экономического взаимодействия и географические параметры. Такие модели, по оценке А. Широва, могут быть удобными, учитывая различные особенности регионов, что делает их более «персонализированными» по географическому положению, к недостаткам относят встречающееся доминирование географических параметров над экономическими [12]. Последнее, на наш взгляд зависит от объективности разработчиков таких моделей, что, в принципе, актуально для всех прогнозных моделей. Такой вид прогнозирования с использованием моделей получил развитие в коммерческом секторе логистики, с использованием специализированного ПО (см. ESRI, IndorGIS, GRASS, AnyLogic и др.). K сожалению, по заданным нами критериям данный метод прогнозирования хотя и более релевантен на методологическом уровне за счет учета пространственных факторов, он так же, как и метод «затраты-выпуск», исключает из анализа экономико-психологические и поведенческие факторы, что недопустимо упрощает саму модель, исключая из нее собственно реакции агентов и их стили поведения.

И метод прогнозирования «затраты-выпуск» и гравитационные модели относятся к типу моделей общего равновесия, поскольку они опираются на базовое представление об экономической системе как о сбалансированной, уравновешенной. 
Модели общего равновесия нередко подвергаются критике со стороны ряда ученых, поскольку такой способ прогнозирования имеет свои ограничения. Так в одной из работ М. Грассини (2009) описал критические вопросы касательно моделей общего равновесия [3]. Основные же тезисы его работы мы выразим четырьмя пунктами:

1) Модели общего равновесия основаны на неоклассической парадигме, что по мнению ряда ученых (в первую очередь поведенческие экономисты, экономические психологи и др.), и самого Грассини, не имеет отношения к реальной экономической среде. Ссылаясь на работы Диксона и Парментера (1996) он говорит о том, что такого рода модели подразделяют экономических агентов разного уровня на максимизаторов прибыли или полезности, в зависимости от цели, но действующих строго рационально. Также, данный тезис подкрепляется цитатой Т. Хаавелмо (1944), о проблеме соответствия эконометрических моделей и действительности, и, наличием разницы между «наблюдаемыми», «истинными» и «теоретическими» переменными [3]. Вместе с тем, А. Широв называет опору на неоклассическую парадигму преимуществом для моделей общего равновесия и динамических межотраслевых моделей [12].

2) Прогнозирование с использованием моделей общего равновесия используется для сравнительного статистического анализа от периода к периоду, причем различие между статичными и динамичными моделями обусловлено наличием параметра $t$, и, если переменная t названа периодом (час, день, год и т.д.), то такие модели Грассини предлагает считать сопоставимыми с шагом итерации в статичной модели.

3) Модели общего равновесия не имеют отношения к реальной экономической среде. Автор считает, что при прогнозировании с использованием таких моделей исследователи не стремятся смоделировать экономическую систему соответствующую реальности, а стремятся изменить реальность под удобные расчеты [3]. Что выражается в создании специальных баз данных для определенных видов расчетов, например, введение системы национальных счетов. 
Касательно этого пункта М. Грассини даже говорит о возможной новой профессии: изготовитель данных для таких моделей.

4) Рассмотрение в модели только относительных цен, несуществующих в реальной жизни и соответственно абстрагированных от реальности [3]. Само по себе наличие относительных цен - это метод сравнения периодов в товарах, например, количестве золота, зерна, нефти и др. Но ученый заостряет внимание в неприменимости такого подхода к реальности, аналогично, на наш взгляд, выглядит такой феномен как денежная иллюзия, выражающая разницу в номинальных и реальных деньгах, при этом применимая к реальности, но лишь после определенных расчетов, как дополнительная переменная расчетов (поправка на изменение цен).

Другая сторона слабости данных методов прогнозирования относительно выделенных нами критериев представлена позицией, выраженной в 1976 году Р. Лукасом. Данный ученый, на наш взгляд, поднимает очень важный вопрос об использовании статичных математических моделей для прогнозирования в экономике: изменения в экономической политике, произошедшие на основе применения данных моделей должны менять и сами модели, поскольку изменились правила взаимодействия [15]. Надо отметить, что данная позиция впервые в качестве методологического условия прогнозирования экономических процессов была высказана в 1920-е гг. выдающимся российским экономистом Н.Д. Кондратьевым, отмечавшим, что само прогнозирование уже влияет на поведение людей и тем самым искажает процессы относительно ситуации, в которой у действующих агентов прогноза нет [4]. Однако, обсуждение критических возражений Р. Лукаса (1976 год) и общего методологического условия любого прогноза в экономике Н.Д. Кондратьева (1926 год) продолжается и в наши дни. Так, реагируя на критику Р. Лукаса касаемо долгосрочных прогнозов и того факта, что при изменении экономической политики могут изменяться взаимосвязи, что ведет к неприменимости модели, А. Широв говорит о том, что такие виды прогноза очень чувствительны к гипотезам относительно параметров технического прогресса, и это делает их очень уязвимыми, с 
другой стороны он выражает несогласие с критикой, поскольку считает долгосрочные прогнозы наиболее интересным и содержательным методом оценки перспективной экономической политики [12]. В целом в ответе на критику, А. Широв отмечает, что в сравнении с краткосрочными и среднесрочными прогнозами долгосрочные имеют большую свободу, но при этом они опираются на определенные «якоря» для более точного прогнозирования (демография, сценарии развития, запасы природных ресурсов и т.д.) [12].

Таким образом, очевидно, что используемые в методах прогнозирования модели общего равновесия пока не позволяют преодолеть эффект влияния самого прогноза и действий на его основе на качество предсказания. На наш взгляд, М. Грассини, достаточно точно описал проблемы, с которыми столкнулись модели общего равновесия и основной из них выступает проблема опоры на неоклассическую экономическую школу, для которой за базовый постулат берется рациональная модель поведения экономических агентов, известная как Homo Economicus, и представляющая фирмы как максимизаторов прибыли, а домохозяйства как максимизаторов полезности. На сегодня накоплен большой объем данных, опровергающих соответствие данной модели действительности (это подтверждают Парадокс Алле и Эффект неоднозначности Эллсберга и др.) [8], но методов прогнозирования, учитывающих достижения поведенческих экономистов и экономических психологов пока не выработано.

Используемые методы прогнозирования развития социально-экономической системы зарекомендовали себя по отдельности в относительно стабильных условиях экономического развития, но в тоже время не дают возможности комплексного разноуровнего прогноза, не дают представления о том, как будут реализовываться изменения на микроуровне. С методологической точки зрения, модели на основе межотраслевого баланса используют математический аппарат и основаны на неоклассической экономической теории, которая в современных исследованиях экономических психологов и поведенческих экономистов показала свое несоответствие реальной экономической среде. В тоже время использование метаматематического 
аппарата возможно с некоторыми корректировками или опорой на другие методы прогнозирования.

Таким образом, в современной экономической науке существует потребность в разработке новых или в модификации существующих методов прогнозирования, способных учесть действие экономикопсихологических и поведенческих факторов. Прежде всего, это касается включения в технологию прогнозирования эффекта трансформации поведения агентов, вызванного наличием самого прогноза.

Работа выполнена в рамках проекта РФФИ № 18-013-00952 «Конкурс проектов 2018 года фундаментальных научных исследований на тему: "Психологические особенности экономической активности личности в условиях совершенной экономики»

This study was carried out as part of the RFBR project No. 18-01300952 "Competition of the projects of 2018 fundamental research on the topic: Psychological features of an individual's economic activity in a perfect economy"

\section{Список литературы}

1. Баранов Э.Ф., Пионтковский Д.И., Старицына Е.А. Методологические проблемы использования метода структурной декомпозиции в модели «Затраты-Выпуск» на современном этапе // Проблемы прогнозирования. 2019. №2. С. 27-36.

2. Большой экономический словарь [Электронный ресурc]. URL: http://economics.niv.ru/doc/dictionary/big-economic/fc/slovar-207-13. htm\#zag-11650 (дата обращения: 14.01.2020).

3. Грассини М. Проблемы применения вычислимых моделей общего равновесия для прогнозирования экономической динамики // Проблемы прогнозирования. 2009. №2. С. 30-48.

4. Кондратьев Н.Д. Большие циклы конъюнктуры и теория предвидения: Избранные труды. М.: Академический проект, 2015. С. 471-518.

5. Леонтьев В.В. и др. Исследования структуры американской экономики / пер. с англ. А.С. Игнтаьева под ред. А.А. Конюса. Москва: Государственное статистическое издательство. 1958. С. 18-22. 
6. Масакова И.Д. Российская практика составления таблиц «ЗатратыВыпуск»: проблемы и перспективы развития // Проблемы прогнозирования. 2019. №2. С. 14-26.

7. Неверов А.Н. Экономико-психологические факторы общественного развития. Саратов: СГСЭУ, 2008.

8. Неверов А. Н., Маркелов А. Ю. Склонность к риску как способность к экономической деятельности: результаты лабораторного эксперимента // Психология способностей и одаренности Материалы всероссийской научно-практической конференции. Под редакцией В.А. Мазилова. Ярославский государственный педагогический университет им. К.Д. Ушинского. 2019. С. 518-521. 2019. C. 409.

9. Суворов А. В. и др. Оценки динамики и структуры человеческого капитала для российской экономики за 1991-2012 гг. // Проблемы прогнозирования. 2015. №. 2. С. 3-15.

10. Толковый словарь Ожегова [Электронный ресурc]. URL: https:// slovarozhegova.ru/word.php?wordid=14661 (дата обращения: 14.01.2020).

11. Шеллинг Т.К. Микромотивы и макроповедение / Пер. с англ. И. Кушнаревой; ред. перевода Д. Шестаков. М.: Изд-во Институт Гайдара, 2016. 344 c.

12. Широв А.А. Роль инструментальных методов анализа и прогнозирования при обосновании экономической политики // Проблемы прогнозирования. 2017. №2. С. 3-9.

13. Широв А.А., Саяпова А.Р., Янтовский А.А. Интегрированный межотраслевой баланс как элемент анализа и прогнозирования связей на постсоветском пространстве // Проблемы прогнозирования. 2015. №1. C. 11-21.

14. Friedman M. The Methodology of Positive Economics. / M.Friedman. Essays in Positive Economics. Chicago: University of Chicago Press, 1953, pp. 3-43.

15. Lucas R. E. et al. Econometric policy evaluation: A critique // CarnegieRochester conference series on Public Policy 1. New York: American Elsevier. 1976. V. 1, pp. 19-46. 


\section{References}

1. Baranov E.F., Piontkovskiy D.I., Staritsyna E.A. Problemy prognozirovaniya. 2019. №2, pp. 27-36.

2. Bol'shoy ekonomicheskiy slovar' [Big economic dictionary]. http://economics.niv.ru/doc/dictionary/big-economic/fc/slovar-207-13.htm\#zag-11650

3. Grassini M. Problemy prognozirovaniya. 2009. №2, pp. 30-48.

4. Kondrat'ev N.D. Bol'shie tsikly kon"yunktury i teoriya predvideniya: Izbrannye trudy [Big cycles of conjuncture and the theory of foresight: Selected works]. M.: Akademicheskiy proekt, 2015, pp. 471-518.

5. Leont'ev V.V. et al. Issledovaniya struktury amerikanskoy ekonomiki [Research of the structure of the American economy] / Per. s angl. A.S. Ignta'eva pod red. A.A. Konyusa. Moskva: Gosudarstvennoe statisticheskoe izdatel'stvo. 1958, pp. 18-22.

6. Masakova I.D. Problemy prognozirovaniya. 2019. №2, pp. 14-26.

7. Neverov A.N. Ekonomiko-psikhologicheskie faktory obshchestvennogo razvitiya [Economic and psychological factors of social development]. Saratov: SGSEU, 2008.

8. Neverov A.N., Markelov A.Yu. Psikhologiya sposobnostey i odarennosti Materialy vserossiyskoy nauchno-prakticheskoy konferentsii [Psychology of abilities and giftedness Materials of the All-Russian scientific-practical conference]. Ed. V.A. Mazilov. Yaroslavl State Pedagogical University named after K. D. Ushinsky. 2019, pp. 518-521. 2019. P. 409.

9. Suvorov A.V. et al. Problemy prognozirovaniya. 2015. №, pp. 3-15.

10. Tolkovyy slovar' Ozhegova [Ozhegov's Explanatory Dictionary]. https:// slovarozhegova.ru/word.php?wordid=14661

11. Shelling T.K. Mikromotivy i makropovedenie [Micromotives and macrobehavior] / Per. s angl. I. Kushnarevoy; red. perevoda D. Shestakov. M.: Izd-vo Institut Gaydara, 2016. 344 p.

12. Shirov A.A. Problemy prognozirovaniya. 2017. №2, pp. 3-9.

13. Shirov A.A., Sayapova A.R., Yantovskiy A.A. Problemy prognozirovaniya. 2015. №1, pp. 11-21.

14. Friedman M. The Methodology of Positive Economics / M. Friedman. Essays in Positive Economics. Chicago: University of Chicago Press, 1953, pp. 3-43. 
15. Lucas R.E. et al. Econometric policy evaluation: A critique. Carnegie-Rochester conference series on Public Policy 1. New York: American Elsevier. 1976. V. 1, pp. 19-46.

\section{ДАННЫЕ ОБ АВТОРЕ}

Голубев Федор Сергеевич, аспирант факультета магистратуры и аспирантуры кафедры экономической психологии и психологии государственной службы, младший научный сотрудник лаборатории экономического поведения Центра психологоэкономических исследований

Поволжский института управления - филиал Российской академии народного хозяйства и государственной службы ул. Московская 164, г. Саратов, Саратовская область, 410012, Российская Федеращия golubev@ipei.ru

\section{DATA ABOUT THE AUTHOR}

Golubev Fedor Sergeevich, Ph.D. student of the department of economic psychology and psychology of public management, junior researcher, Labor of Economic Behavior of the Center of Psycho-Economic Research

The Saratov Volga Regional Institute of Administration - branch of the Russian Academy of National Economy and Public Administration

164, Moskovskaya str. Saratov, Saratov region, 410012, Russian Federation

golubev@ipei.ru

SPIN-code: 3555-5278

ORCID: 0000-0002-5726-5574 